\title{
Sexually Reproducing Cellular Automata *
}

\author{
PAUL M. B. VITÁNYI \\ Mathematisch Centrum, Amsterdam, The Netherlands \\ Communicated by Gordon Pask
}

\begin{abstract}
Sexual reproduction is modeled and investigated in the formal framework of John von Neumann's theory of self-reproducing cellular automata. It is argued that the transition from asexual to sexual reproduction necessitates a change in number and structure of the genetic tapes involved. To an asexually reproducing cellular automaton only one genetic tape is attached, viz. the description which enables the automaton to construct cell for cell a replica of itself. The sexually reproducing automaton, however, must possess two, nearly identical, genetic tapes of a deviating structure, i.e. programs partitioned into sections embodying the various construction and behavioral algorithms to be executed. It is shown that the recombination of the parents' characteristics in the offspring closely conforms to recombination in nature. Similarities and differences with biological systems are discussed.
\end{abstract}

\section{INTRODUCTION}

Abstract automata are information processing discrete parameter systems and may be viewed as mathematical models for natural automata (e.g. biological organisms, solar systems) and artificial automata (e.g. computers, slot machines). Excellent textbooks in automata theory have been published $[11,5]$.

To study the logical intricacies of machine self-reproduction, von Neumann introduced in about 1953 the notion of a cellular automaton [21]. In general terms, a cellular automaton consists of a finite aggregate of interacting automata and is said to reproduce if it constructs a replica of itself. This process clearly constitutes asexual reproduction: the offspring is an exact copy of a single parent. The aim of the present article is to model and to investigate sexual reproduction in cellular automata theory. As the terminology in use is apt to create confusion, we wish to clarify some matters at the outset.

* This article is based on a 1969 term paper composed under supervision of Prof. L. A. M. Verbeek, Technological University of Delft.

(C) American Elsevier Publishing Company, Inc., 1973 
- One cellular automaton consists of several interacting automata called cells.

- The self-reproduction of a cellular automaton should be taken as a model for the reproduction of a single natural cell rather than as a model for the reproduction of a multicellular organism.

- When the reproduction of a multicellular organism is to be modeled, we should employ an aggregate of cellular automata, and hence an aggregate of aggregates of cells, as in Sec. 4.

-Arbib [4] and others view the cellular automaton as corresponding to a multicellular organism, and a cell of the cellular automaton as corresponding to a cell in that organism. However, as Arbib points out, there are a lot of dissimilarities between automata reproduction and organism reproduction in this case.

-We sometimes use "automaton" for "cellular automaton" when no confusion can result.

\subsection{MOTIVATION AND BACKGROUND}

Biological methodology usually consists of description and classification according to actual observations. There are important exceptions to this: the Darwinian theory of evolution and the Mendelian theory of heredity. "But in between these two regions, especially in the area of growth and development, there is very little except systematic observation and description, and most theorizing is tied closely to the observed biological facts" (Ref. 2, pp. 3-4). In a paper on a model of self-reproduction Stahl (Ref. 19, p. 68) holds that "the logical primitives of a valid model must correspond to genes and enzymes, and that all the major entities (DNA's, RNA's, enzymes, proteins, organelles, ATP, and cell walls) and functions (DNA copying, RNA copying, ribosome action, enzyme action, passive physiochemical action, and aggregation or polymerization) of real cells should somehow be represented symbolically". A computer simulation of living cells which conforms to this dictum is described by Weinberg and his coauthors $[8,23,25]$. On the other hand, even in an abstract system of self-reproduction, bearing almost no relationship to biochemistry, interesting theorems about the logical requirements and limitations of self-reproduction may be obtained. ${ }^{1}$ Von Neumann suggested that automata theory should include a study of the way in

${ }^{1}$ The historical development of geometry from "self-evident" axioms to "logically possible" axioms may serve to illustrate the differences between the two approaches. Euclidean geometry describes and formalizes empirical observations. On the other hand, e.g., nonEuclidean geometry derives necessary consequences from a (consistent) set of axioms. Comparison of the two approaches provides a better insight into the nature of geometry. 
which initial information serves to regulate growth and change in the structure of an automaton. He noted that we associate with machines used for construction a certain degenerating tendency; we expect a machine to build a machine of less complexity. However, when organisms reproduce, we expect their offspring to be of a complexity at least equal (evolution!) to that of the parent. In view of this apparent conflict von Neumann [21] introduced cellular automata as a logical framework in which to study the problem of how to make machines reproduce themselves in a purely mechanical fashion, as a way of throwing light on some fundamental problems of biology, and as a problem concerning the capabilities and limitations of machines. (See Ref. 5, pp. 349-350 and Ref. 13, p. 17). Aside from this abstract approach there exist interesting machine models of reproduction such as the mechanical "tilt blocks" model of Penrose [16], the electromechanical "toy-train" model of Jacobson [9], and the electromagnetic "relais" model of Morowitz [14].

Let us consider machines composed from some suitable collection of elementary parts. We may choose these components to be self-reproductive and so remove the problem or consider it at a descriptive level. We may also choose components which are very simple (not self-reproducing, few different states, etc.), but aggregates of which can be self-reproducing. This still leaves trivial cases like crystallization and the Penrose model. To avoid triviality, we shall require that the machines are capable of doing something meaningful beside reproducing. Therefore we need the notion of a Turing machine [20]. A Turing machine is a logical device consisting of a reading head that travels about an indefinitely expandable tape that is divided into squares. The reading head is under control of a finite program that determines at each step, according to the symbol in the tape square under scan, whether this symbol should be overprinted by another symbol and whether the reading head should move one square left or right. When presented with an argument on its tape such a machine computes the value of a particular function for that argument. For every function that "intuitively" can be computed there exists such a Turing machine (Turing's hypothesis). It has been shown $[20,11]$ that a Turing machine can be constructed which, given an appropriately coded description of any other Turing machine on its tape, will imitate the behavior of that machine towards an argument written elsewhere on the same tape. Hence such a Universal Turing machine is capable of computing every computable function (equivalently, executing every algorithm) if supplied with an appropriate description of a corresponding Turing machine. We shall consider the reproduction of machines which simulate Universal Turing machines as meaningful self-reproduction. The demonstration of such a machine proceeds as follows. 
(i) Exhibition of a Universal Constructor (UC) which can build any one of a large class of constructible machines drawing from an infinite supply of a fixed number of different components. The UC produces a machine when furnished with a complete description, represented by a coded chain of the said elements. The UC is also shown to be a Universal Computer, i.e. Universal Turing machine simulator, using such a chain as an indefinitely expandable tape.

(ii) This Universal Computer-Constructor (UCC) appears to be a constructible machine, and, by the following slight modification, a selfreproducing one. To the parent machine is attached a component for component description of the machine itself in passive state: the genetic tape. ${ }^{2}$ The parent constructs another machine according to the genetic tape, copies the tape, and attaches the copy at the appropriate place of the constructed machine. Subsequently, the offspring is activated and separated from its parent. Hence this machine is a self-reproducing UCC.

The procedures outlined above can be implemented and executed in a cellular space $[21,7]$.

In reproductive processes as described above the offspring is an exact copy of a single parent: asexual reproduction. When we look for a common denominator in the manifold variations which evolution uses to express reproduction, we find as central theme, an alternating cycle where two haploid cells (single chromosome set) merge to one diploid cell (double chromosome set), the zygote. This zygote produces directly or via intermediate steps new haploid reproducing cells which inherit the presumably regrouped parental chromosomes and genes. These recombinations are tested as to their usefulness against the environment. From the viewpoint of evolution this process works much more efficiently than that of just mutations. Strangely enough, notwithstanding recent remarkable advances in molecular biology, practically nothing is known about the physiology of sexual processes. In this light it seems worthwhile, for reasons mentioned before, to investigate the sexual reproduction of machines.

Let us imagine that several (two) machines cooperate and supply the genetic material for one offspring machine. By assigning diverse reproductive processes to specialized types of machines we obtain (two) sexes. The recombination of the parents' characteristics in the offspring shall be shown to correspond closely to nature. Hence we shall construct by our method a formal model of sexual reproduction which yields the

${ }^{2}$ The constructed machine should be passive during the construction phase, so as not to interfere with the construction activities going on. Furthermore, while it is possible and desirable that a machine has only one passive state, it has of necessity a very large number of active states. 
familiar properties of heredity not by treating them as given a priori, but by deriving them indirectly from certain logical assumptions.

\subsection{SELF-REPRODUCING CELLULAR AUTOMATA}

Our investigations are conducted in the framework of a cellular space in which we abstract from problems of kinematics, energy, and, more or less, geometry. We may think of a cellular space as an infinite chess board, each square or cell of which can be in any one of a finite number of cellular states symbolizing the presence in that cell of one out of a finite set of different components. If a component $X$ is able to occur in different states $x_{1}, \ldots, x_{n}$ (e.g. a memory element) we assign a different cellular state $q_{x_{i}}$ to each $x_{i}$. Let $\phi$ denote the set of cellular states. Then each

$$
q \in\left\{q_{x_{1}}, \ldots, q_{x_{n}}\right\}=\phi_{X} \subseteq \phi
$$

indicates the presence of component $X$ in a certain state in the cell. The quiescent state $0 \in \phi$ will symbolize the absence of any component in a cell. Therefore, an aggregate of finitely many interacting components can be represented by an aggregate of finitely many interacting nonquiescent cells.

Cellular automata were introduced by von Neumann [21]. General properties of cellular spaces were studied in e.g. Refs. 13 and 18. As a starting point and a reference frame for our discussion of sexually reproducing automata we use the self-reproducing automata of Codd [7]. We review Codd's model in a cursory manner below, so as to make this article accessible to readers not acquainted with cellular automata theory.

For our purpose a cellular space consists of an infinite 2-dimensional plane divided into unit squares, each of which represents a copy of a single finite-state automaton or cell and is designated by its integer coordinates $(x, y) \in I \times I$. Each cell can be in any one of a finite set of cellular states $\phi=\{0,1,2, \ldots, n\}$, and all cells change their states simultaneously in discrete time steps. Each cell has associated with it a neighborhood (Fig. 1), consisting of the cell itself and its four immediate nondiagonal neighbors, and a local transition function $f: \phi^{5} \rightarrow \phi$. The next state of a cell is given by $f(C N E S W)=R$ where the capitals stand for the Current state, the states of the North, East, South, and West neighbor, and the Resulting state, respectively. A configuration $c: I \times I \rightarrow \phi$ is an assignment of states to each cell in the space. At time $t=0$ the initial configuration $c_{0}$ is given. The global transition function $F$ (simultaneous invocation of $f$ in each neighborhood of the cellular space) determines a sequence of configurations

$$
c_{0}, c_{1}, c_{2}, \ldots, c_{t}, \ldots
$$


where

Thus

$$
c_{t+1}=F\left(c_{t}\right)=F^{t+1}\left(c_{0}\right) \text { for all times } t
$$

$$
F(c)(x, y)=f(C N E S W),
$$

where $C, N, E, S$, and $W$ represent the states of $(x, y)$ and its four neighbors under $c$.

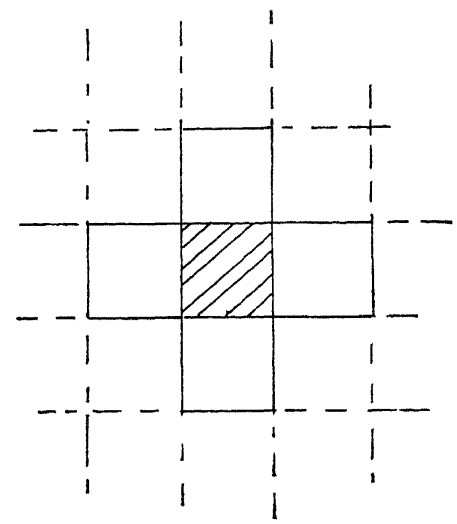

Fig. 1. Cell and neighbors.

To ensure the effective computability of $c_{t}$ for all times $t$, the following two restrictions are stated.

(i) There exists a quiescent state $q \in \phi$ such that $f(q q q q q)=q$, i.e. a quiescent cell with quiescent neighbors remains quiescent.

(ii) At time $t=0$,

$$
\{(x, y) \mid c(x, y) \neq q\}
$$

is a finite set. We shall take $q=0$.

COROLLARY

For all times $t \geqslant 0$,

$$
\{(x, y) \mid c(x, y) \neq 0\}
$$

is a finite set, the support of $c$ denoted by $\sup (c)$.

A configuration $c^{\prime}$ is a subconfiguration of $c$ if $c^{\prime}\left|\sup \left(c^{\prime}\right)=c\right| \sup \left(c^{\prime}\right)$. A configuration $c$ is passive if $F(c)=c$, and completely passive if every subconfiguration of $c$ is passive. By disjoint configurations $c$ and $d$ we mean that their supports are disjoint. The union $c \cup d$ of $c$ and $d$ is defined by

if $c$ and $d$ are disjoint.

$$
(c \cup d)(x, y)= \begin{cases}c(x, y) & \text { if }(x, y) \in \sup (c), \\ d(x, y) & \text { if }(x, y) \in \sup (d), \\ 0 & \text { otherwise }\end{cases}
$$


If $W \subseteq \phi$ and $c: I \times I \rightarrow W$ then $c$ is a configuration over alphabet $W$. If every $c: I \times I \rightarrow W$ is (completely) passive, $W$ is called a (completely) passive set. In our considerations $\{0,1\}$ is a completely passive set, i.e. every configuration which assigns only states 0 or 1 to cells in the space is completely passive. We call such a configuration a $(0,1)$-configuration.

Usually, the term "configuration" will be used loosely to mean $c \mid \sup (c)$. The devices of subconfiguration and union of configurations allow us to talk about parts of the over-all configuration of the cellular space. For instance, when $c \cup d$ contains the $(0,1)$-configuration $d, d$ does not change unless there is a time $t$ such that

$$
F^{t}(c \cup d)\left|\sup \left(F^{t}(d)\right) \neq F^{t}(d)\right| \sup \left(F^{t}(d)\right),
$$

i.e. $c$ is an adjacent disjoint configuration and passes information to $d$. When no confusion can result " $\sup (c)$ " is sometimes used to refer to a specific region of the cellular space more or less (but for some quiescent cells) demarcated by $\sup (c)$. Under suitable interpretation we can prove the following theorems which will be used extensively in the sequel.

\section{THEOREM 1}

(Von Neumann [21], Codd [7]). For every Turing machine there exists an, initially completely passive, configuration in the cellular space which simulates it, using a linear string of cells in state 0 or state 1 as the indefinitely expandable (binary) tape on which the computation is performed.

\section{COROLLARY}

There exists a configuration in the cellular space which simulates a Universal Turing machine.

REMARK 1

Such a computing configuration, or a configuration that contains one, is called a cellular automaton. Usually, it consists of a network of paths embedded in the cellular space. These paths are arrays of cells in state 1 (completely passive configurations), along which signals in the form of propagating sequences of cellular states can be transmitted. ${ }^{3}$ A typical sequence is " $0 s$ " such that $s \in\{4,5, \ldots, n\}$ leads and 0 trails (Appendix B). Special configurations act as, among others, junctions, fan-ins and fanouts of signals (signals merge and multiply), signal transformers (all

${ }^{3}$ Actually, due to some technical considerations the paths are coated or sheathed with a layer of cells in state 2 on both sides (see Appendix B). This is attained by injecting a signal (henceforth subsumed under the activating signals) which propagates throughout the entire network of paths, sheathing each path it traverses. The structure which results, a network of sheathed paths, is a configuration over $\{0,1,2\}$ and is passive, but not completely passive. 
signals can be derived from a single "activating" signal), periodic emitters (clock pulse), and logical elements, thus supplying all the essentials with which the logical structure of an information processing device can be embedded in the cellular space. A cellular automaton interacts with its cellular space environment, e.g. reads and writes 0's and 1's on its tape, by means of a constructing arm. A constructing arm is a path, extended out from the automaton into a quiescent area, along which signals are transmitted extending the path (left, right, and straight ahead by annexing the appropriate 0 cells at the end), retracting the path (left, right, and straight backwards by returning the appropriate end cells to 0), sensing whether the cell next to the end cell is in state 0 or state 1 , and changing that state from 0 to 1 or from 1 to 0 . Some examples can be found in Appendix B.

A constructing arm enables a cellular automaton to reach out and read, write, and erase every $(0,1)$-configuration anywhere in the cellular space. As a cellular automaton consists, nearly enough, of a signal carrying structure of $(0,1)$-configurations, in particular such a structure can be constructed by sweeping a constructing arm column by column row after row over a quiescent construction site while changing the appropriate cells to state 1 . When the completely passive structure of an automaton has thus been laid out, some activating signals are injected at an appropriate entry point (the injection receiver), and subsequently the constructing arm is separated from the newly constructed automaton. It is advantageous to consider only initially completely passive automata, as they will not interfere with construction activities while under construction. Let us consider as constructible machines all cellular automata which can be constructed in this way, e.g. all Turing machine simulators of Theorem 1.

THEOREM 2

(Von Neumann, Codd). There exists a configuration in the cellular space called a Universal Computer-Constructor (UCC) with the following properties.

(i) For every Turing machine $\mathbf{T}$, there exists a coded description $B(\mathbf{T})$ such that when $B(T)$ is placed on the program tape attached to the UCC, the UCC will simulate $\mathbf{T}$ on an attached data tape.

(ii) For each constructible machine $\mathbf{M}$, there is a coded description $D(\mathbf{M})$ of $\mathbf{M}$ such that, when $D(\mathbf{M})$ is placed on the program tape attached to the UCC, the UCC will construct M (Fig. 2).

\section{REMARK 2}

The UCC reads its instructions, e.g., for the cell by cell construction of the completely passive structure of an offspring, in 4-bit words from 
a linear $(0,1)$-configuration or program tape by means of one constructing arm, and proceeds to execute these instructions by sending sequences of signals along another constructing arm towards a data tape or a construction site (Fig. 2). The set of 4-bit instructions interpretable and executable by the UCC consists of

(i) a computation-universal instruction set equivalent to the instruction set of the program machine version [22] of a Universal Turing machine,

(ii) a construction-universal instruction set, e.g., extend (constructing arm), extend left, extend right, retract, retract left, retract right (hence the constructing arm can reach out to, and retract from, any cell in the cellular space),

(iii) some instructions to switch the input to, and output from, the interpreting and executive sections of the automaton from one constructing arm to another, and

(iv) some instructions for the injection of the activating signals and a stop instruction.

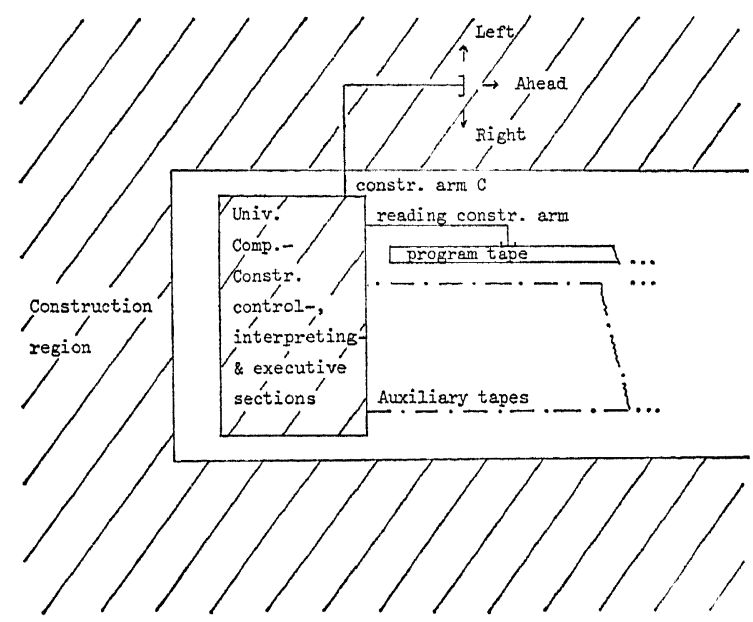

FIG. 2. The Universal Computer-Constructor (UCC) and its construction region.

The instruction set as described consists of 14 elements and can be coded in 4-bit words. It enables the UCC to compute any Turing-computable function from $C^{*}$ into $C^{*}$, where $C^{*}$ is the set of all $(0,1)$-configurations that do nor intersect with the area occupied by the machine. Whether the UCC computes on a linear $(0,1)$-configuration or constructs and activates a constructible machine depends on its program tape. The UCC is a constructible machine in the defined sense. When it is furnished with 
$D$ (UCC), i.e., the description of its own completely passive structure coded in 4-bit words on its program tape, it constructs a duplicate of this structure. When, furthermore, the UCC copies its program tape at the appropriate location and proceeds to inject the activating signals, selfreproduction has been attained (Fig. 3).

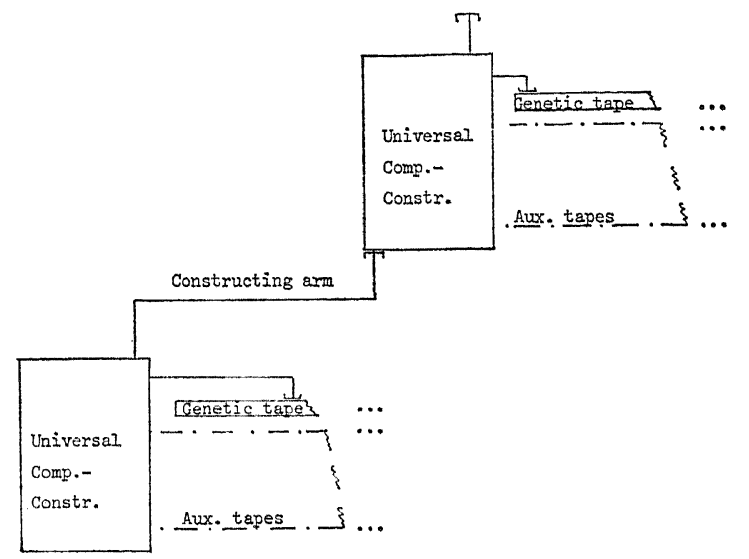

FIG. 3. Self-reproduction of the Universal Computer-Constructor.

THEOREM 3

(Von Neumann, Codd). There exists a configuration in the cellular space which is a self-reproducing UCC.

REMARK 3

Directed by its program tape this machine is able to

(i) construct, read, and erase any member of $C^{*}$,

(ii) compute any Turing-computable function from $C^{*}$ into $C^{*}$.

(iii) construct a replica of itself at every location in the cellular space, copy the program tape containing the self-description $D$ (UCC), and activate the offspring. When the program tape contains such a self-description it is called the genetic tape.

The study of self-reproduction of cellular automata has been exclusively concerned with asexual reproduction, i.e. one automaton constructs another according to its own genetic material, possibly with computed minor variants (Refs. 15 and 3, see also Ref. 6). In the present article sexual reproduction of cellular automata is investigated, incidentally providing a first formal model of natural sexual reproduction. As mentioned before, the sexuality lies in the fact that two (or more) automata take part in the construction of one offspring and contribute to its genetic 
material. More than two automata per offspring can be considered, but this only complicates the picture and does not contribute to the advantages of the model. As a matter of fact, "nature" seems to be of the same opinion; many species have more than two sexes (mating types), but two parent reproduction is the only one known in nature. By our deligation of the necessary diverse algorithms to two specialized types, we obtain one obviously male (M-type) and one obviously female (F-type) cellular automaton sex. Incorporation of all necessary algorithms in each automaton would yield "hermaphrodite" automata capable of "crossfertilization."

By considering populations of sexually reproducing cellular automata and introducing chance mutations in the genetic material, we shall be able to detect familiar notions (of sexual reproduction), such as "species of automata," "genetic pool," "evolutionary variability," "recombination," etc. Various aspects of sexual reproduction can be (formally) studied by means of our model, e.g.

infertility among seemingly compatible species (depending on the difference in instruction code and interpreting mechanism used by these species),

Mendelian laws,

sterile hybrids and sterility coupled to sex (inheritable by means of the other sex),

mating types, and

sex-linked inheritance.

\section{DESCRIPTION OF THE MODEL}

Mainly, the sexually reproducing automaton consists of the body $B$, two genetic tapes $T_{1}$ and $T_{2}$ containing the encoded construction and behavioral algorithms (cf. 2.4), constructing arms $C$ (both $\mathbf{M -}$ and F-type) and $D$ (only $\mathbf{F}$-type) to execute these algorithms, and reading-writing constructing arms $R_{1}, R_{2}, R_{3}$, and $R_{4}$ to read from $T_{1}$ and $T_{2}$ (Fig. 4). Furthermore, there are some auxiliary tapes and reading-writing constructing arms which are not of interest here (cf. Codd, Ref. 7). The two specialized types or sexes result from our aim to simplify the individual automata by a delegation of the tasks that have to be performed, e.g. searching for the other automaton (M-type), contributing genetic material (M- and F-type), construction of the offspring (F-type).

Prior to the construction of the offspring we need its genetic material (if it is redundant also a clearly determined part of it constituting a complete description) according to which the new automaton is to be constructed, since we have to know in advance which characteristics of what 
parent will be incorporated in the offspring. More specifically, we want the description of the offspring to be unambiguously extractable from its total genetic material. Because each automaton has two parents and due to the above and considerations set forth in Sec. 2.4 , every automaton possesses two (complete) genetic tapes.

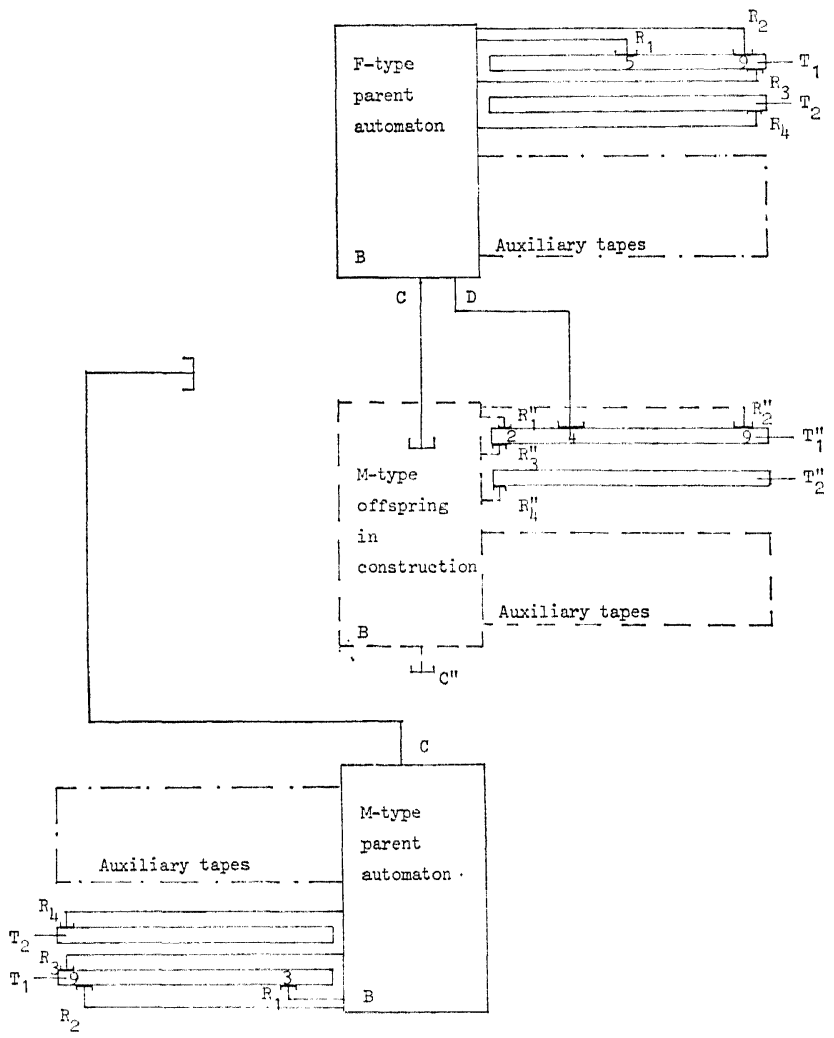

FIG. 4. Sexually reproducing cellular automata.

The F-type parent constructs the offspring according to the $T_{1}^{\prime \prime}$ genetic tape and subsequently activates the completely passive offspring by the injection of activating signals, separating the constructing arm $C$ in the process. We are somehow reminded of birth and cutting the umbilical cord. (Incidentally, automata existing in the cellular space at time 0 may possess only one genetic tape, viz. $T_{1}$, and thus be haploid.)

The above represents a necessary departure from the usual (asexual) automata reproduction practice where first the offspring is constructed by the parent according to the parental genetic tape, and afterwards is 
supplied with a copy of this tape. Observe that similarly in natural sexual reproduction (of higher organisms) first the genetic material of the offspring is constructed and then the organism corresponding to the genetics, while in natural asexual reproduction (e.g. mitosis) first the cellular material is increased and then the genetic material duplicated.

The recombination of the parents' characteristics in the offspring is due to the procedures used to convey the genetic material from the parents to the offspring (Fig. 5). By means of a random copying procedure, i.e. Sec. 2.1, each parent maps its two genetic tapes onto one initial tape image; $\mathbf{M}$ and $\mathbf{F}$ produce $T_{1}^{\prime}$ and $T_{2}^{\prime}$, respectively. Subsequently, each dominant word or characteristic (Appendix A) that has a recessive counterpart is placed on $T_{1}^{\prime}$ while the other is placed on $T_{2}^{\prime}$; if both are of the same kind then the distribution is random (cf. Sec. 2.2.3). The definitive tape images $T_{1}^{\prime \prime}$ and $T_{2}^{\prime \prime}$ result from this process, and it is from $T_{1}^{\prime \prime}$ that the offspring is constructed.

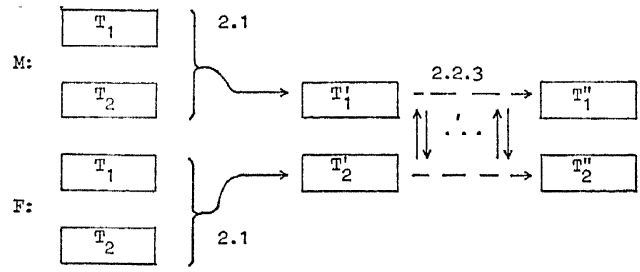

FIG. 5. Schematic representation of genetic recombination processes.

\subsection{COPYING OF THE GENETIC MATERIAL}

The genetic material is transmitted from each parent to the offspring by means of a copying procedure which maps the two parental tapes onto one initial tape image by choosing the $n$th word on it randomly from between the $n$th word on $T_{1}$ and the $n$th word on $T_{2} . T_{1}$ and $T_{2}$ are read by $R_{3}$ and $R_{4}$, and the initial tape image is constructed by $C$. If one of the genetic tapes $T_{1}$ and $T_{2}$ is longer than the other, the copying automaton chooses the word on the longer tape. If both $T_{1}$ and $T_{2}$ contain the all-0 word, consisting of only quiescent states, in the $n$th position, the copying procedure is terminated. The randomness mentioned above may be obtained from the computation of a random number by the computationuniversal part of the automaton or some other means.

\subsection{THE M-ALGORITHM}

In the course of its reproductive behavior the M-automaton executes the following algorithm (see also Fig. 6): 


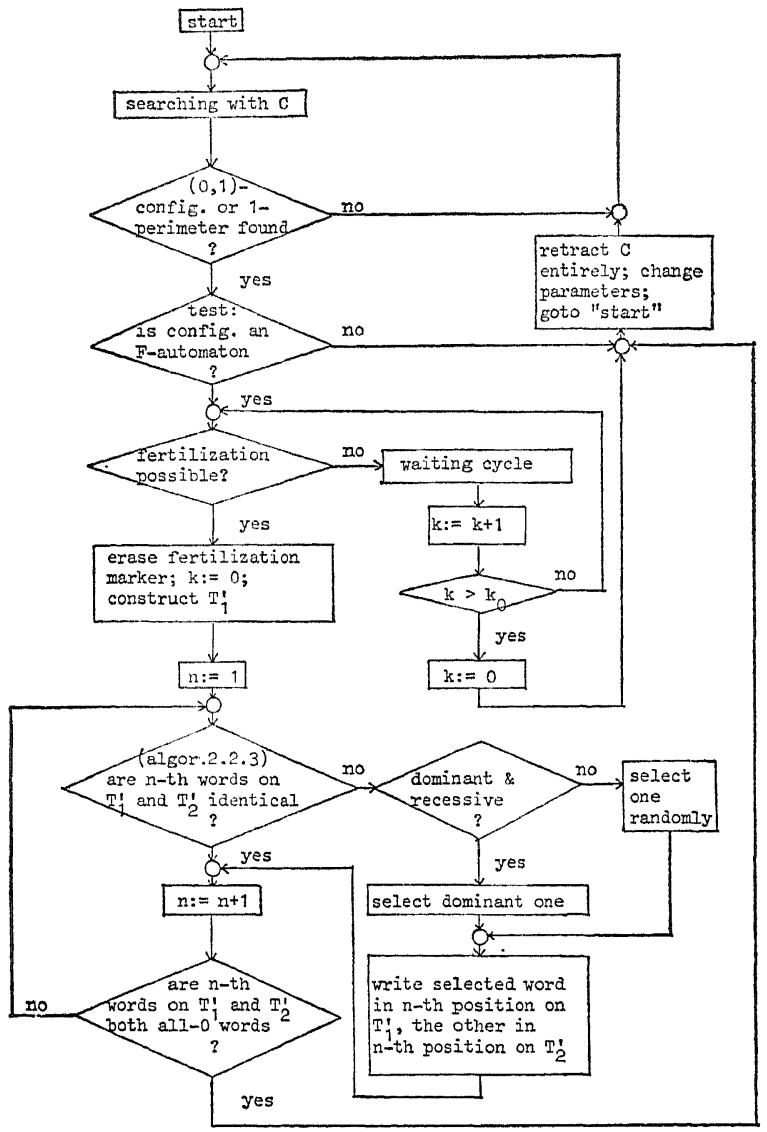

FIG. 6. Flowchart M-algorithm.

\subsubsection{Searching}

(i) The $\mathbf{M}$-automaton takes the initiative in the reproductive process It searches, systematically or at random, and in both cases governed by its computation-universal part, the cellular space with $C$ until it finds an object. Such an object may be a $(0,1)$-configuration or a configuration surrounded by a perimeter of cells in state 1 (see Appendix A). Objects consisting of other configurations may cause unspecified behavior or dying of the $\mathbf{M}$-automaton.

(ii) $\mathbf{M}$ ascertains, according to some well-defined characteristics (Appendix A), whether or not the object found is an F-automaton. If so $\mathbf{M}$ does (iii), otherwise (xi).

(iii) $\mathbf{M}$ ascertains whether fertilization is possible, e.g. by reading a 
special word or fertilization marker on a specific corner of the 1-perimeter or "skin" of the $\mathbf{F}$-automaton (Appendix A). If so, $\mathbf{M}$ does (vii), otherwise (iv).

(iv) $\mathbf{M}$ completes a waiting cycle of a certain length.

(v) $\mathbf{M}$ checks the fertilization marker. If fertilization still is not possible, $\mathbf{M}$ does (vi), otherwise $\mathbf{M}$ resets a counter $K$ to zero and does (vii).

(vi) $\mathbf{M}$ increments $K$ with 1 and ascertains whether the contents of the counter surpass a bound $k_{0}$. If not, $\mathbf{M}$ does (iv), otherwise $\mathbf{M}$ resets $K$ to zero and does (xi).

\subsubsection{Fertilization}

(vii) $M$ changes the fertilization marker from "fertile" to "infertile," e.g. by erasing it. $\mathbf{M}$ copies (cf. Sec. 2.1) its genetic tapes such that sup(tape image) relative to sup(fertilization marker) is situated as indicated by, or computed from, the fertilization marker. After the mixing process of Sec. 2.2.3 this tape image-or rather the one then present on the same support-will constitute the genetic tape $T_{1}^{\prime \prime}$ from the offspring-to-be (cf. Fig. 4).

\subsubsection{Mixing of the Genetic Material}

(viii) If the $\mathbf{F}$-automaton is fertilization prone, the $T_{2}^{\prime}$ initial tape image, i.e. the precursor of $T_{2}^{\prime \prime}$ (cf. Sec. 2.2.2), of the future offspring already has been constructed, viz. in Sec. 2.3. By means of constructing $\operatorname{arm} C$ the M-automaton now reads, remembers, and compares successively the words in identical positions on the previously constructed $T_{1}^{\prime}$ and $T_{2}^{\prime}$. If they are identical nonquiescent words, $\mathbf{M}$ resumes (viii) with the next pair of words, otherwise $\mathbf{M}$ executes (ix).

(ix) According to an extra mark bit per word, $M$ checks on the dominant or recessive property of each of both words (cf. also Appendix A).

(x) If both words are of the same kind, $\mathbf{M}$ randomly writes one of them in the considered word position of $T_{1}^{\prime}$ and the other in the considered word position of $T_{2}^{\prime}$. If one of the words is dominant and the other is recessive, $\mathbf{M}$ writes the dominant word in the considered word position of $T_{1}^{\prime}$ and the recessive one in the considered word position of $T_{2}^{\prime}$. If $T_{1}^{\prime}$ is longer than $T_{2}^{\prime}$, or vice versa, the choice is between the all-0 word and a nonquiescent word. The latter is written in the appropriate position of $T_{1}^{\prime}$, the former in the appropriate position of $T_{2}^{\prime}$. Is the choice between two all- 0 words, the end of both tapes has been reached and $\mathbf{M}$ executes (xi). Otherwise, $\mathbf{M}$ starts again at (viii) with the next pair of words.

(xi) $\mathbf{M}$ retracts constructing $\operatorname{arm} C$.

(xii) $\mathbf{M}$ changes some parameters in the search procedure and starts again at (i). 


\section{REMARK 1}

Subsequent to the completion of Sec. 2.2.3 the constructed genetic tapes have attained their definitive form, and the $T_{1}^{\prime \prime}$ tape of the offspringto-be contains all the inherited dominant traits (or factors) that had recessive counterparts.

\subsection{THE F-ALGORITHM}

In the course of its reproductive behavior, the $\mathbf{F}$-automaton executes the following algorithm (see also Fig. 7):

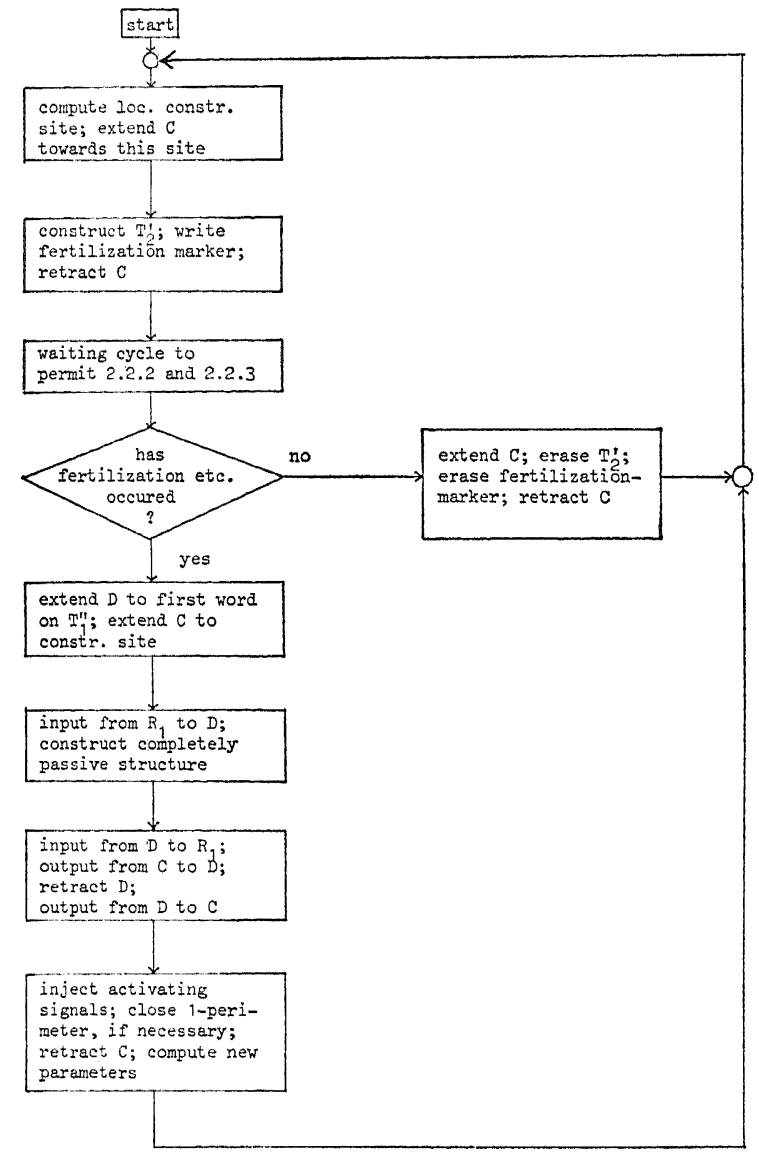

FIG. 7. Flowchart F-algorithm.

(i) From some parameters, $\mathbf{F}$ computes the location of a construction site relative to $\sup (\mathbf{F})$, and extends constructing arm $C$ towards this location. 
(ii) $\mathbf{F}$ copies (see Sec. 2.1) its genetic tapes at the appropriate place computed in (i). After execution of Sec. 2.2.3 by some M-automaton this tape image-or rather the one then present on the same support-will constitute the $T_{2}^{\prime \prime}$ genetic tape of the future offspring.

(iii) $\mathbf{F}$ writes the fertilization marker (cf. Sec. 2.2.1) at the appropriate corner of its "skin". This fertilization marker must be a $(0,1)$-configuration that contains the information from which $\mathbf{M}$ can compute the relative location to sup(fertilization marker) where $\mathbf{M}$ executes Secs. 2.2.2 and 2.2.3.

(iv) $\mathbf{F}$ retracts constructing arm $C$.

(v) $\mathbf{F}$ executes a waiting cycle of a specified length during which some $\mathbf{M}$ can execute Secs. 2.2.2 and 2.2.3.

(vi) $\mathbf{F}$ extends constructing arm $C$ towards its fertilization marker and checks whether fertilization has occurred; if some $\mathbf{M}$ has executed Secs. 2.2.2 and 2.2.3, the fertilization marker is on "infertile," e.g. has been erased. If so, $\mathbf{F}$ does (viii), otherwise $\mathbf{F}$ changes the fertilization marker from "fertile" to "infertile" and does (vii).

(vii) $\mathrm{F}$ erases the tape image $T_{2}^{\prime}$, retracts constructing $\operatorname{arm} C$, and starts again at (i).

(viii) Until now F's effectuating organ has been constructing arm $C$, i.e. the output signal sequences are routed along $C$. Now $\mathbf{F}$ reroutes the output signal sequences along $D$ and extends $D$ towards the first word in $T_{1}^{\prime \prime}$.

(ix) F reroutes the output again along $C$ and extends $C$ towards the appropriate construction site relative to $\sup \left(T_{1}^{\prime \prime}\right)$.

(x) At this stage $\mathbf{F}$ starts with the construction of the $(0,1)$-configuration or completely passive structure of the offspring. In order to do this, the input to $\mathbf{F}$ is switched from $R_{1}$ to $D$ (see Fig. 4), and $\mathbf{F}$ proceeds to execute the construction specifications laid down in the description of the future offspring on the genetic tape $T_{1}^{\prime \prime}$.

(xi) When (x) is completed, the input to $F$ is switched back from $D$ to $R_{1}$. The output from $\mathbf{F}$ is rerouted along $D, D$ is retracted, and the output is switched back again to constructing $\operatorname{arm} C$.

(xii) $\mathbf{F}$ injects the activating signals in the completely passive offspring via $C$, thus separating $C$ from the offspring, and closes the 1-perimeter (if present) over the injection receiver of the offspring.

(xiii) $\mathbf{F}$ retracts $C$, computes new parameters for the ones used in (i) and starts again at (i).

REMARK 2

After construction of the completely passive offspring, the readingwriting constructing arm $R_{2}^{\prime \prime}$ (Fig. 4) is placed on the first word of sec. 9 of $T_{1}^{\prime \prime}$, i.e. the section containing the instructions for the nonreproductive 
behavior of the newly constructed offspring (cf. Sec. 2.4). This may consist in the computation of a certain computable function (on one of the auxiliary tapes) or something similar.

\section{REMARK 3}

The activating signals of (xii) also initialize the reading processes by $R_{1}^{\prime \prime}$ and $R_{2}^{\prime \prime}$, and in so doing start the reproductive and nonreproductive behavior of the new automaton (cf. Sec. 2.4). To be able to conduct two demeanors simultaneously, each automaton must contain at least a computation-universal and a construction-universal part. However, this presents no difficulties in principle.

\section{REMARK 4}

When we examine algorithms 2.2 and 2.3 , we clearly discern periodic fertility in the $\mathbf{F}$-automaton, during which fertilization by an $\mathbf{M}$-automaton is possible. An alternative would have been to halt the $\mathbf{F}$-automaton after the copying process and have it activated again by the $\mathbf{M}$-automaton subsequent to the genetic mixing.

\subsection{THE GENETIC TAPE}

The structure and number of genetic tapes involved in sexual reproduction is based on certain logical considerations. We require that:

(i) An automaton's genetic material is composed from random contributions of both parents and contains a complete self-description,

(ii) the genetic material is furnished prior to construction, and

(iii) the offspring is constructed, by the F-type parent, from a permanently retraceable description.

For (i)-(iii) one genetic tape per automaton suffices, e.g. the F-automaton constructs a tape copy with random "gaps" consisting of all-1 words (the all- 0 word is already in use to indicate the end of tape) subsequently to be substituted by an M-automaton with words copied from its tape.

Obviously, for such a process to work and produce the genetics for a well-formed offspring, it is necessary that the genetic tapes of both automata are similar with respect to structure, instruction sequences, and the diverse algorithms. By similar we mean here that, although instructions in identical positions on the tapes may be different, an interchanging of (sequences of) them will not render the algorithms involved incoherent, and meaningless.

This kind of sexuality, however, reminds one of the rudimentary sexual 
processes (generation renewal) of bacteria and lacks the principal evolutionary purpose of sexual variations, the ensurance of a retaining and a periodically detaching of recessive mutations in a population. For such "higher" forms of sexual reproduction we need at least two genetic tapes per automaton, each of which is a mixture of two parental tapes. A tape image is composed by randomly taking successive instructions from one or the other of the parent's tapes. The observation about similarity of tapes also holds here, each parent must have two similar tapes. Therefore both tapes of an automaton have to be complete, i.e. contain all (reproductive) behavioral and construction algorithms in a fixed order. Every unlike mechanical procedure of composing a tape image of two dissimilar tapes would entail an almost impossibly difficult administration. As the genetic constitution (self-description) of the offspring must be retraceable subsequent to construction, the offspring cannot be constructed by taking instructions successively, according to the dominant property and randomly if of the same kind, from one or the other initial tape image. Consequently, we first produce the definitive tape image $T_{1}^{\prime \prime}$ (by means of Sec. 2.2.3) containing the complete description of the offspring.

Furthermore, (iii) is required so that the $\mathrm{F}$-automaton can construct the offspring from a single genetic tape. (iii) emphasizes again the necessity for similar tapes.

Obviously, the F-automaton has to start its construction job with a fixed instruction on $T_{1}^{\prime \prime}$, e.g. the first one. As each tape contains all algorithms, the first instruction must select the tape section containing the description of the specific part of the offspring's sexual type, i.e. the part of the configuration (of body $B$ ) that is different for $\mathbf{M}$ and $\mathbf{F}$. After construction, the offspring's $R_{1}^{\prime \prime}$ starts reading the second instruction directing it to the behavioral algorithm suited to its sex. Hence we require, in contrast with the asexual case, a tape partitioned into behavioral and construction sections. Note that there is a marked difference between the construction sections (e.g. secs. 4, 6, and 7 below) from which the "physical lay-out" of the automaton is constructed, and the more algorithmic sections (e.g. secs. 3 and 5 below) which govern the behavior to be performed; these latter sections are read, interpreted, and executed by the configuration constructed according to the former sections.

In asexual reproduction no distinction is made between the different tape sections, as the problem of different sexes and behavior does not arise, i.e. the automaton computes a location, proceeds to execute the construction sequence, copies the tape, and activates the offspring. These four different actions are accomplished by using different interpreting sections in sequence; the behavior is built into the automaton more or less as hardware subroutines in an electronic computer. The complicated 
nature of sexual reproduction, however, necessitates special behavioral and construction algorithms and hence tape sections, thus accentuating differences and similarities between construction and behavior as embedded in a cellular space.

Note. From Sec. 2.3, we see that in sexual reproduction the activating procedure is not a part of the "hardware" of the automaton or of a construction algorithm as in the asexual case, but a part of behavioral algorithm 2.3.

A genetic tape is composed of 9 sections, numbered 1 to 9 (Fig. 8). Each section contains a sequence of binary coded instructions either embodying a behavioral algorithm or a construction algorithm. Sections 1 and 2 determine whether the automaton is M-type of F-type. These sections play the part of the $\mathrm{X}$ and $\mathrm{Y}$ chromosome in biology.

\begin{tabular}{|c|c|c|c|c|c|c|c|c|} 
sex & \multicolumn{3}{c}{ M-type } & \multicolumn{3}{c}{ F-type } & \multicolumn{3}{c}{ collective } & individual \\
\hline 1 & 12 & 3 & 4 & 5 & 6 & 7 & 8 & 9 \\
\hline
\end{tabular}

FIG. 8. The genetic tape.

Section 1. A jump, i.e. transfer of the head of the reading constructing arm to a designated instruction word on the tape, to section 4 if the genetic tape determines an $\mathbf{M}$-automaton, a jump to section 6 if it determines an F-automaton.

Section 2. A jump to section 3 if the tape determines an $\mathbf{M}$-automaton, to section 5 if it determines an $\mathbf{F}$-automaton.

Section 3. A subprogram that embodies algorithm 2.2. Note that when the genetic tape determines an $\mathbf{M}$-automaton, section 2 may also consist of empty instructions since section 3 follows immediately.

Section 4. The construction sequence for the construction of the specific reproductive part of the completely passive M-automaton. The last instruction is a jump to section 7 .

Section 5. A subprogram that embodies algorithm 2.3.

Section 6. As section 4, with $\mathbf{F}$ substituted for $\mathbf{M}$.

Section 7. The construction sequence for the construction of the identical part of the M- and $\mathbf{F}$-automaton.

Section 8. The construction sequence for the construction of an individual part of the completely passive automaton. The last instruction on 8 gives the control back to section 5 of the constructing $\mathbf{F}$-type parent's $T_{1}$.

Section 9. Instructions for the individual nonreproductive behavior of the automaton, as mentioned in Remark 2. These instructions may be read, interpreted, and executed by the part of the automaton that is specified in section 8 . 
When the instruction code or the partitioning of the genetic tape is different for two automata, we may talk about different species of automata. Usually, within a species, sections 1 to 7 of the genetic tapes $T_{1}$ and $T_{2}$ will be identical.

Section 1 and section 2 of $T_{2}$ always specify jumps to section 6 and section 5, respectively, i.e. $T_{2}$ always is $\mathrm{X}$-type. In an $\mathbf{F}$-automaton, both $T_{1}$ and $T_{2}$ are $\mathbf{X}$-type. In an $\mathbf{M}$-automaton $T_{2}$ is $\mathbf{X}$-type while $T_{1}$ is $\mathbf{Y}$-type, i.e. section 1 and section 2 specify jumps to section 4 and section 3 , respectively. Because an automaton is constructed according to its $T_{1}$ genetic tape (cf. Sec. 2.3) this tape controls the type, or sex, of the automaton.

In the first instance (before the mixing process 2.2.3), $T_{2}^{\prime}$ is an image (cf. Sec. 2.1) of the genetic tapes of the F-automaton and therefore is $\mathrm{X}$-type: $T_{1}^{\prime}$ is an initial tape image of the genetic tapes of the $\mathbf{M}$-automaton and is $\mathrm{X}$ - or $\mathrm{Y}$-type on a random basis. We also attach a dominant bit to the instruction words of sections 1 and 2 if they specify jumps to sections 4 and 3 , respectively, and a recessive bit if they specify jumps to sections 6 and 5. Then if $T_{1}^{\prime}$ is $\mathbf{Y}$-type, sections 1 and 2, consisting of dominant instructions, are unchanged by the mixing of the genetic material.

Note that every genetic tape carries the potential for the development of both an F-type and an $\mathbf{M}$-type automaton. Which one is realized depends on the instructions at the commencement of the tape, and the interpreting apparatus of the F-type parent. Such a mechanism may take different forms; appearing from the medley of sex chromosome mechanisms known in biology, "nature" seems of a similar opinion (cf. the case of the Protenor Belfragei in Sec. 4 [1, 12]).

\section{AUTOMATA GENETICS}

In the remainder of this article we shall often use a more or less anthropomorphic terminology without implying, however, that the analogies perceived rest on similarities with actual biochemical processes. Rather, the cellular automaton analogues should be taken to furnish abstract and illuminating (cf. Sec. 1.1) counterparts of natural phenomena. The sketchy outline of our model of sexual reproduction given above allows much latitude in form and behavior of a pair of automata of different sexes with a compatible fertilization technique. With respect to the recombination of the parents characteristics in the offspring we can distinguish between the following cases.

(a) The two parents use a different binary coding for identical instructions. In this circumstance, the mixing phase 2.2 .3 will scramble the instructions on the offspring's genetic tapes in such a way that the constructing automaton will construct a meaningless configuration, if any. 
It even seems possible that the $\mathrm{F}$-automaton will die by destroying vital parts of itself. Thus, two automata that use different instruction codes can have no progeny, notwithstanding the fact that they may consist of correct compatible configurations and have a compatible fertilization technique (no fertility among seemingly compatible, but genetically different, species).

(b) The parents use the same instruction code, but the partitioning of their respective genetic tapes differs with respect to positioning and/or length of one or more of the sections 1-7. Again, this will result in meaningless parts of the offspring's genetic tapes, viz. the part after the first difference, causing faulty parts of the offspring and/or senseless behavior following activation. If one of the initial tape images $T_{1}^{\prime}$ and $T_{2}^{\prime}$ contains only dominant factors and the other only recessive ones, the effects described in (a) and (b) will not occur. Nevertheless, the offspring will be infertile owing to 2.2 .2 or 2.3 (ii), which make use of 2.1, and so disform the initial tape images of the offspring's direct progeny. As in (d-v) we are reminded of sterile hybrids.

(c) The parents use the same instruction code, and the initial tape images $T_{1}^{\prime}$ and $T_{2}^{\prime}$ differ with respect to length and/or positioning only in sections 8 and 9 , the individual traits. The result is an offspring, wellformed with respect to reproducing abilities, with far-going variations in the nonreproductive part of the configuration and its nonreproductive behavior. (c) is an extreme form of (d-vi).

(d) The parents use the same instruction code and partitioning, but the initial tape images $T_{1}^{\prime}$ and $T_{2}^{\prime}$ differ with respect to the instructions in one or more of the sections 3-9. The difference occurs between the following.

(i) Sections 3. In an M-type offspring the configuration that regulates its reproductive behavior will be in good order but reproduction impossible since algorithm 2.2 is deranged. The phenomenon is reminiscent of behavioral, or psychically induced, impotence. An F-type offspring will be fertile with respect to M-type automata of its parents' species. The effect described then is dormant, but will exhibit itself in future M-type progeny of the F-type offspring.

(ii) Sections 4. In an M-type offspring the configuration that regulates the reproductive behavior will not function as intended. A sterile, or organically impotent, M-type individual appears. An F-type offspring will be fertile with respect to M-type automata of its parents' species, but the effect described is dormant and will exhibit itself in future M-type progeny.

(iii) Sections 5. The effect is analogous to the one described in (i) with the roles of the $\mathbf{M}$ - and $\mathbf{F}$-types interchanged. The defect in the F-type progeny may be termed "frigidity." 
(iv) Sections 6. The effect is analogous to the one described in (ii) with the roles of the $\mathbf{M}$ - and $\mathbf{F}$-types interchanged.

(v) Sections 7. This may cause a disfigurement in the reproducing part of the offspring's configuration regardless whether it be $\mathbf{M}$ - or $\mathbf{F}$-type. We are reminded of sterile hybrids. If section 7 contains a description for a computation-universal part of the configuration, not indispensable for reproduction (e.g. for the generation of random numbers), the distortion of this configuration causes a defect in the offspring's behavior, which we may compare with an inheritable mental illness due to an organic defect.

(vi) Sections 8 and/or 9. We assume that this usually holds in a species of our automata; it is meant to convey individual traits to different automata, respectively physical (viz. qua configuration) and behavioral (viz. qua performed nonreproductive algorithm); it implies the existence of a population of genetically different individuals of sexually reproducing cellular automata for which notions like "genetic pool," "evolution" and "adaptability," "evolutionary variability," etc. are appropriate.

The species of an automaton is determined by the instruction code and the partitioning of the genetic tapes with respect to length and/or positioning of sections $1-7$. Case $(\mathrm{d}-\mathrm{v})$ includes the possibility of sterile hybrids. We may consider cases (d-i) and (d-iii) as psychical or behavioral derangements which obstruct the procreating activities of one sex and are inheritable by means of the other sex. If the automata we have been dealing with would have to perform other activities besides procreation and computation, (d-v) could well impair the viability of these automata.

Mutations can be brought into the model in an obvious way by suitable changes in the genetic tapes resulting in, for instance, the consequences mentioned above, viz. a change in one of the genetic tapes of a parent gives via the copying procedure the effects as treated. Apart from the disadvantageous outcomes already indicated, advantageous ones may also be generated by a mutation. A beneficial change in the progeny can be brought about by tentative small changes in the genetic tapes of populations of automata, such that the cumulative effect of a set of these changes incorporated in one automaton in the course of the sexual reproduction processes promotes its viability. Assume a certain redundancy in tape structure to the effect that the change of one word on a genetic tape need not have fatal consequences. We then may observe a transition of varicties of automata, i.e. classes of automata, differing in important respects qua sections 1 to 7 but not qua instruction code, which are still reconcilable with respect to reproduction, into different species of automata using an identical instruction code but not reconcilable with respect to reproduction. We obtain a universe populated with different species of automata 
using the same instruction code (assuming that they all stem from the same stock). Whether this observation has a biological interpretation cannot be judged here.

Sex-linked inheritance is introduced easily, e.g. by enlarging sections 4 and 6 with a nonreproductive part. A small difference in the additional part of section 4 of the genetic tape will (possibly) cause the M-type progeny to have corresponding (nonreproductive but sex-linked) traits; not so the F-type progeny in which the trait remains dormant but may be passed on to future generations. A biological analog is constituted by hemophilia. If we take color-blindness to be a behavioral defect, we can apply similar considerations with respect to section 3 (or similarly section 5) of the genetic tape.

A change in the last instructions of sections 4 or 6 of $T_{1}$, such that no jumps to section 7 but rather to sections 6 or 4 , respectively, are performed, produces something like "hermaphrodite" automata in the offspring. These automata may display either the M-type or F-type reproductive conduct.

When an $\mathbf{F}$-automaton erroneously has its reading constructing arm $R_{1}$ placed on section 3 of $T_{1}$ or, vice versa an M-automaton its $R_{1}$ on section 5 of $T_{1}$, e.g. by a wrong jump in section 2 , the resulting behavior reminds one of transsexuality. Protagonists of the theory might see their conviction confirmed that transsexuality is not a functional (in this context, acquired in life) phenomenon, but a genetically induced one.

\section{COMPARISON WITH NATURAL SYSTEMS}

Roughly speaking, the genetic tape corresponds to a set of chromosomes. A comparison between automata reproduction and organism replication shows that (cf. Ref. 4, pp. 150-151):

(i) each cell of a multicellular organism contains one or more (e.g. two) sets of chromosomes, whereas in our automata the (two) genetic tapes are attached to the cellular automaton as a whole, and

(ii) as noted, automaton reproduction depends on the passivity of the constituent elements. Any subassembly remains passive until the whole structure is complete. In biological development interaction between subassemblies of the growing embryo presumably plays an essential role.

These apparent contrasts can more or less be removed by a modification of our model, such that each cellular automaton corresponds to one cell of a multicellular organism, and an aggregate of interacting cellular automata corresponds to a whole multicellular organism. Assume that we append to each genetic tape a section 10 and a section 11 . 
Section 10 contains the construction algorithm for an additional UCC in each automaton.

Section 11 serves to store information relevant to the history of the automaton ancestors of the automaton concerned (this section will not be copied by process 2.1 ).

When the additional UCC of an automaton is activated it proceeds to build a replica of the automaton according to $T_{1}$, duplicates $T_{1}$ and $T_{2}$, including the (modified) contents of section 11 then present, at the appropriate location of the replica, and passes control to section 9 . This section contains an algorithm, executable by the configuration built according to section 8 , which governs the interaction with other automata of an aggregate. Under control of section 9 the automaton, according to information received, modifies section 11 and, determined by the new contents of this section, may proceed to build another replica at a computed location.

Such a mitotic cellular automaton is taken to correspond to one natural cell and the interacting aggregate constitutes a developmental model of a multicellular organism. Different contents of section 11 cause different reactions to other automata, constituting our analog of cell-differentiation. At a certain stage, determined by the information then contained in section 11 , some automata of the aggregate may pursue their original purpose, i.e. reproduce sexually with automata of different sex of an adjacent aggregate in a similar stage. Subsequent to sexual reproduction, section 11 of the new automaton contains only all-0 words, viz. it is not copied. The new offspring starts replicating itself in the asexual fashion described above, thus starting a new aggregate, the cycle repeats.

Note that all cellular automata of an aggregate are genetically identical but for their sections 11 (cell-differentiation) and genetical accidents (e.g. mutation, cancer), and hence they all contain the building plan for the same aggregate and determine the same sex. In our developmental model we meet abstract counterparts of, e.g., fertilized egg-cells, celldifferentiation, sexual maturity and immaturity, and genotypically different cells in the same organism as an exceptional case.

The model is consistent with the "axioms" of development presented by Apter [2] and reviewed critically by Arbib (Ref. 4, pp. 131-132).

(1) The unit of development is a cell (i.e. cellular automaton). Information is not contained merely in the genetic "instructions" but also in the cytoplasmic "processors." In our model, automata of the same species interpret the same instruction code, i.e. the structure of an automaton contains information. 
(2) All cells in the organism are genotypically identical. Except for cell-differentiation (i.e. differences with respect to section 11) or genetical accidents, our model accords. We might speculate that owing to the introduction of behavioral sections on the tape, the sexually reproducing automata are much better equipped for ontogenic (individual) learning than the traditional asexually reproducing ones, which are but suited to phylogenetic (racial) learning. The voiding of section 11 during sexual reproduction can be interpreted as the nontransmittance of lifetime learning during this kind of reproduction.

(3) An organism develops through the self-reproduction of the cells composing it.

(4) Intercommunication between cells is a prerequisite for coherent development. This does not seem to be a logically necessary requirement, since an organism may develop in a "monadic" fashion, i.e. clockwork synchronization suggesting interaction, as is indicated by, e.g., Ref. 17.

(5) An organism controls the important aspects of its own development. This subsumes the problem of cell reproduction. In our model we see a synthesis: cell reproduction is simulated by asexual automata reproduction; the cells of the cellular space correspond to macromolecules and development is simulated by an aggregate of cellular automata.

In an important way an asexually reproducing population, stemming from the same stock, can be viewed as a single organism, the identities of the different individuals being often not very distinctive. In a sexually reproducing population the distinction between different individuals is very clear even in a formal way. A new individual is created only by sexual reproduction, i.e. by mixing the genetic material especially with respect to sections 8 and 9.

Our model may seem complicated, but owing to the computationconstruction universality of the cellular space, is theoretically feasible. A theory originally proposed by Weissmann [24] and strongly worded by Apter (Ref. 2, p. 157) to the effect that "germ cells reproduce themselves, and, as a side-effect each one has the potentiality of developing into a larger and more complex version of itself" appears natural in the light of our model. It also constitutes a rationale for our point of view in Secs. 1-3 where we were interested in the properties of sexual reproduction of single cellular automata (and not aggregates of them) and the accompanying genetics.

Present biological views concerning sex, growth, and genetics as, e.g., expressed by Mittwoch [12] seem to endorse an (abstract) validity and explaining power of our model. For instance, sex seems to be determined by a chromosome region of a different order of magnitude than 
those that are responsible for Mendelian characteristics. In sex determination the chromosome region involved is likely to be equivalent to a large number of Mendelian genes. In the model, sex is selected by the XY mechanism which activates the chosen parts of the genetic tape; this mechanism, translated into biochemical terms, is of the kind that can be verified or refuted by experiments.

An interesting question seems to be which chromosomes (i.e. tape sections) are necessary and/or sufficient, and what variations in the buildup of the genetic tapes are possible.

As an example let us take the case of the Protenor Belfragei (an insect) $x o(\delta): x x(f)$, i.e. the male possesses an unpaired $X$ chromosome while the female has a pair of homologous $X$ chromosomes. Let sections 1 and 2 of $T_{1}^{\prime}$ contain all-0 words, and let the mixing process be such that initial all-0 words are taken to be dominant. Afterwards, the all-0 word is interpreted by the constructing $\mathbf{F}$-automaton as a "jump to section 4" and by a constructed M-automaton as a "go to next instruction." Verification shows that the sexual reproduction works exemplary when, for this species, $T_{1}$ of the M-type contains all-0 sections 1 and 2 . These and other types of sexual chromosome mechanisms can be investigated by using models with appropriate changes in tapes and interpreting apparatus. We end this section with a few miscellaneous remarks.

(a) Previously, the construction of the offspring was executed by the $\mathbf{F}$-type parent. We may consider the case where the $\mathbf{F}$-automaton constructs an UCC alongside the tape copy in 2.3 , which UCC is fertilized and activated by an $\mathbf{M}$-automaton in 2.2.3. Such a mode of reproduction is reminiscent of that of fishes.

(b) An abstract counterpart for the changing of sexes (e.g. oysters) is supplied by furnishing every automaton with both an M-type and an F-type reproductive part, and having these automata execute in turn 2.2 and 2.3 .

(c) A counterpart for cross-fertilization is provided by the simultaneous execution of 2.2 and 2.3 by two hermaphrodite automata.

(d) Abstract parthenogenesis emerges when an UCC of (a) constructs an offspring with identical $T$ and $T_{2}^{\prime \prime}$ in absence of fertilization.

(e) Abstract mating types are obtained by expanding the tapes with checking conditions, and by manipulating the effects of the copying procedure and 2.2 .3 so as to make identical or different "sexes" sterile towards one another.

(f) We may abolish computational activities and derive a "lower species" or, alternatively, we may do the same for one sex and obtain a sex only capable of reproductive behavior (e.g. honey bees). 
(g) With some fantasy we may think of models of sexual reproduction that have no counterparts in nature.

(h) In all our models of sexual reproduction the concept of "genetic pool" is present. No gene ever is lost, except by destruction of an automaton or a mutation. The dominant and recessive factors have their well-known attributes, e.g. a (momentary) (dis)advantageous trait can be masked by a dominant trait and later in the progeny reveal itself; also dominant traits may be mutated to recessive ones and vice versa.

(i) We may think of mutations as being caused by "cosmic rays" consisting of small moving configurations in the cellular space which change instructions on a genetic tape when they hit one.

\section{CONCLUSION}

Our method of modeling sexual reproduction in the formal framework of a cellular space may prove a useful tool to compare, illuminate, analyze, and classify different models, methods, properties, and anomalies of sexual reproduction, especially with respect to the genetic aspects. Moreover, it provides a first formal (nondescriptive) model for sexual reproduction, may have bearing on behavioral genetics and (the relevant essentials being translated into appropriate biochemical terms) may be experimentally verified or refuted in whole or in parts.

\section{APPENDIX A}

Some objections may be raised against the feasibility of the algorithms 2.2 and 2.3 in Codd's 8-state 5-neighbor cellular space. The most important ones, together with their solutions are reviewed below.

(i) Automata in Codd's space can only read $(0,1)$-configurations and are coated (sheathed) with cells in state 2 . We may introduce additional states to solve this problem, but the easiest way seems to surround every F-automaton with a perimeter of cells in state 1. Such a 1-perimeter also embraces the tapes and reading-writing equipment (Fig. 9). However, the automaton must be able to extend $C$ and $D$. This is accomplished by erasing two sections of 7 cells in the 1-perimeter. The presented solution leaves some events undefined, e.g. when a constructing arm $C$ of an M-automaton touches (reads) another M-automaton or constructing arm. We define such events as mortal to both automata concerned.

(ii) When an $\mathbf{M}$-automaton senses an $\mathbf{F}$-automaton, at regularly spaced intervals along the 1-perimeter, specially coded $(0,1)$-words are situated which identify the F-automaton as such and also furnish the M-automaton with information where to find the fertilization marker. This marker is a particular code word on the 1-perimeter which indicates 


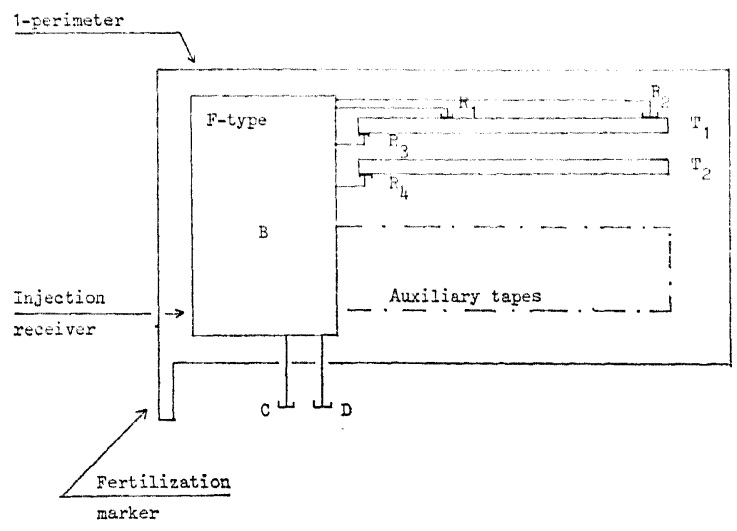

FIG. 9. F-automaton surrounded by 1-perimeter.

whether fertilization is possible, and if so, where in the cellular space the appropriate conduct of $\mathbf{M}$ should take place. The fertilization marker is altered by the constructing arms $C$ of $\mathbf{M}$ and $\mathbf{F}$ at the moments determined by 2.2 and 2.3 .

(iii) The dominant or recessive property of a word on the genetic tape is indicated by the state of an additional cell (mark bit) per word, e.g. dominant $=1$ and recessive $=0$.

\section{APPENDIX B}

Owing to some technical considerations a path or constructing arm, consisting of cells in state 1 , along which a signal $0 s(s \in\{4,5,6,7\})$ travels, is coated or sheathed with cells in state 2 (for details consult Codd's reterence). Examples of signal propagation along a sheathed path and extension of a constructing arm are given below.

According to Sec. 1.2 the local transition function $f: \phi^{5} \rightarrow \phi$ with $f(C N E S W)=R$ determines the next state of a cell. Moreover, let the value of $f$ be invariant under cyclic permutation of the last four arguments; then the given set of transitions governs the propagation of a signal down a linear sheathed path (Fig. 10). The reader may derive from the figures the additional transitions governing the other examples. (Figs 11-14).

$$
\begin{array}{lll}
f(00002)=0 & f(012 s 2)=1 & f(20202)=2 \\
f(s 0212)=0 & f(10212)=1 & f(20212)=2 \\
& f(11212)=1 & f(202 s 2)=2 \\
& & f(112 s 2)=s .
\end{array}
$$




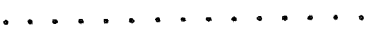

222222222222222

111111051111111

Time Step T

222222222222222

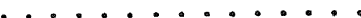

222222222222222

$11111110 \mathrm{~s} 111111$

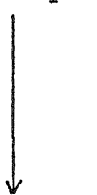

222222222222222

FIG. 10. Propagation down linear sheathed path. The numeral 0 and middle dot $\cdot$ both represent state 0 .

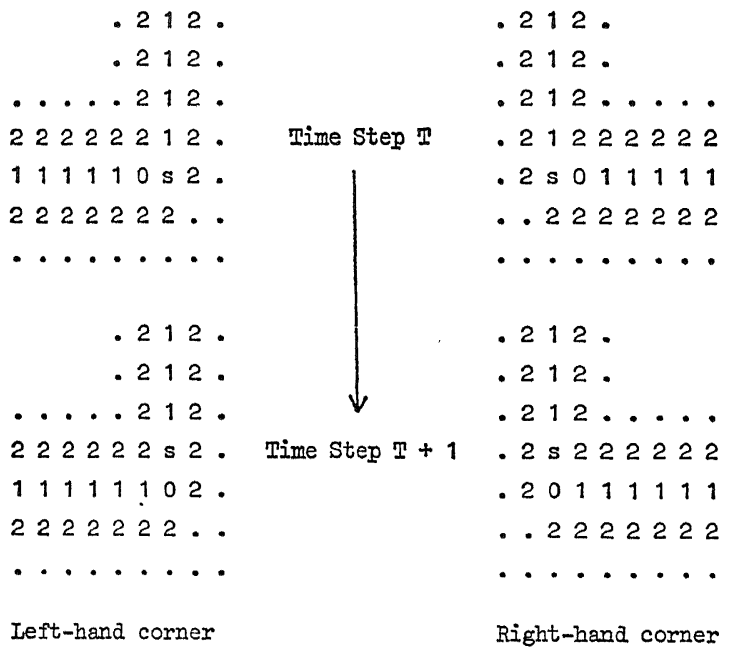

FIG. 11. Signals cornering.

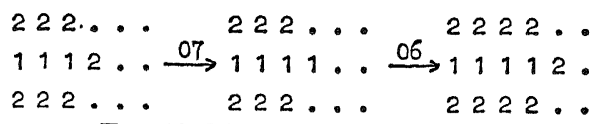

FIG. 12. The extend operation. 


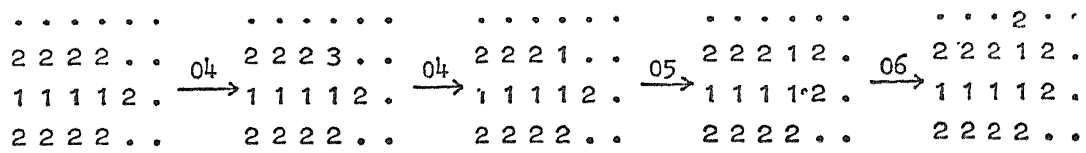

FIG. 13. The extend left operation.

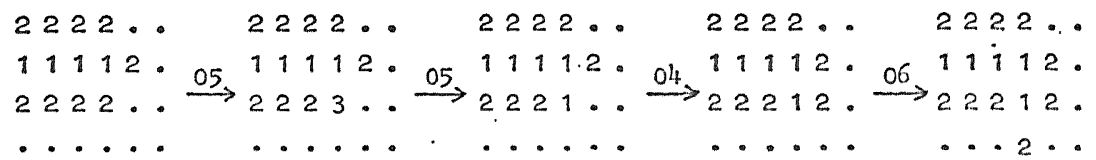

FIG. 14. The extend right operation.

I am indebted to Prof. A. Lindenmayer, Utrecht University, for his encouragement and to Dr. G. Rozenberg for drawing my attention to an independent result of Laing [10] who also considers a notion of sexual reproduction in Von Neumann type cellular spaces; also to Dr. J. W. de Bakker and Mr. P. van Emde Boas, Mathematical Center, for their valuable criticism.

\section{REFERENCES}

1 A. Allison (Ed.), The Biology of Sex, Penguin, New York (1967).

2 M. J. Apter, Cybernetics and Development, Pergamon, New York (1966).

3 M. A. Arbib, A simple self-reproducing universal automaton, Inform. Contr. 9, 177-189 (1966).

4 M. A. Arbib, Automata theory and development: Part I, J. Theoret. Biol. 14, $131-156$ (1967).

5 M. A. Arbib, Theories of Abstract Automata, Prentice Hall, Englewood Cliffs, N.J. (1969).

$6 \mathrm{~J}$. Case, A note on degrees of self-describing Turing machines, $J$. ACM 18, 329-338 (1971).

7 E. F. Codd, Cellular Automata, ACM Monograph Series, Academic, New York (1968).

8 E. D. Goodman, R. Weinberg, and R. A. Laing, A Cell Space Embedding of Simulated Living Cells, Tech. Rept. 03296-1 T, Univ. of Michigan, Ann Arbor (1970).

9 H. Jacobson, On models of reproduction, Am. Scientist 46, 255-284 (1958).

10 R. A. Laing, Asexual and Sexual Reproduction Expressed in the Von Neumann Cellular System (Formalisms for Living Systems (Part I), Secs. 9.2, 9.3 and 9.4). Tech. Rept. 01252 3-T, Univ. of Michigan, Ann Arbor (1970).

11 M. L. Minsky, Computation: Fïnite and Infinite Machine's, Prentice Hall, Englewood Cliffs, N.J. (1967).

12 U. Mittwoch, Sex, growth and chromosomes, New Scientist Sci. J. 51, 126 128 (1971).

13 E. F. Moore, Machine models of self-reproduction, in Math. Prob. Biol. Sci., Proc. Symp. Appl. Math., Vol. 14, Am. Math. Soc., Providence, R.1. (1962), pp. 1733.

14 H. J. Morowitz, A model of reproduction A note, Am. Scicmist 47, $26126.3(1959)$.

$15 \mathrm{~J}$. Myhill, Abstract theory of self-reproduction, in Views on General sistems Theory (M. D. Mesarovic, Ed.), Wiley, New York (1964), pp. 106 118. 
16 L. S. Penrose, Self-reproducing machines, Sci. Am. 200, 105-118, 202 (1959).

17 Chr. P. Raven and J. J. Bezem, Computer simulation of embryonic development I \& II, Proc. Kon. Ned. Akad. v. Wetensch. ser. C 74, 209-223 (1971).

18 A. R. Smith III, Cellular Automata Theory, Tech. Rept. 2, Digital Systems Lab., Stanford Univ., Stanford (1970).

19 W. R. Stahl, A model of self-reproduction based on string-processing finite automata, in Natural Automata and Useful Simulations, Proc. Symp. Fundamental Biol. Models (E. Edelsack, L. Fein, H. Pattee, and A. Callahan, Eds.), Spartan, Washington D.C. (1966), pp. 43-72.

20 A. M. Turing, On computable numbers with an application to the Entscheidungsproblem, Proc. Lond. Math. Soc. 42, 230-265 (1936).

$21 \mathrm{~J}$. von Neumann, The Theory of Self-Reproducing Automata (edited and completed by A. W. Burks). Univ. of Illinois Press, Urbana, Ill. (1966).

$22 \mathrm{H}$. Wang, A variant to Turing's theory of computing machines, J. ACM 4, 63-92 (1957).

23 R. Wein berg and M. Berkus, Bio-Med. Comput. 2, 95-120, 167-188 (1971).

24 A. Weissmann, The continuity of germ plasm as the foundation of a theory of heredity, reprinted in Great Experiments in Biology (G. L. Mordecai et al., Eds.). Prentice Hall, New York (1955).

25 B. P. Zeigler and R. Weinberg, System theoretic analysis of models: computer simulation of a living cell, J. Theoret. Biol. 29, 35-56 (1970). 